\title{
MORT EN CIME DU BOULEAU JAUNE CAUSEE PAR LE CHAMPIGNON NECTRIA GALLIGENA BRES.

\author{
par MARCEL LORTIE ${ }^{1}$
}

\begin{abstract}
RESUME
En plus de causer des chancres typiques sur le tronc et les branches principales des arbres feuillus, le champignon Nectria galligena Bres. peut provoquer la mort de la cime du bouleau jaune grâce au développement de nombreux petits chancres sur les branches et de lésions qui annellent les rameaux. Vus du sol, les arbres ainsi affectés peuvent avoir une mortalité qui va jusqu'à 30 ou 40 p. 100 de la cime et leur aspect général ressemble à celui d'arbres atteints de dépérissement.
\end{abstract}

\section{ABSTRACT}

The inciting agent for typical cankers on the stem and main branches of hardwood trees, the fungus Nectria galligena Bres., may be responsible also for a crown dying of yellow birch owing to the development of numerous small cankers on branches and lesions without calluses which girdle twigs. From the ground, trees that are so affected may show a dying up to 30 or 40 percent of the crown and they look much like trees suffering from dieback.

INTRODUCTION

Chez les arbres feuillus, l'activité du champignon Nectria galligena Bres. se manifeste ordinairement en la formation d'un chancre ellipsoïde sur le tronc ou sur les branches. Une fois que les tissus corticaux autour du point d'infection sont envahis, la zone d'infection s'étend progressivement et le centre se déprime. L'écorce ainsi tuée peut adhérer au bois sousjacent, mais éventuellement elle se brise ou se détache exposant une plaie chancreuse où apparaissent une série de bourrelets concentriques autour d'un point qui garde souvent les traces de la branche où a débuté la maladie. Le chancre peut, en progressant, anneler la tige affectée et ainsi entrainer la mort de la portion distale.

Certains chercheurs en Europe (Viennot-Bourgin, 1949) et en Amérique (Zeller, 1926) ont rapporté que sur les arbres fruitiers, le $N$. galligena peut causer un autre type de dommages, à savoir, le dépérissement des pommiers et des poiriers à la suite de l'infection de rameaux et de petites branches. Dans la forêt, Kienholz et Bidwell (1938) ont rapporté la mort en cime de certains bouleaux jaunes (Betula alleghaniensis Britt.) par suite de la présence de petits chancres probablement nectriens qui avaient annelé des rameaux.

\section{OBSERVATIONS}

En 1959, dans la forêt de Duchesnay, comté de Portneuf, près de Québec, 34 arbres avaient fait le sujet d'une étude particulière concernant l'importance du chancre nectrien. De ce nombre, 2 arbres portaient un bon nombre de branches et de rameaux affectés de petits chancres ou de lésions sans callus qui avaient annelé la tige porteuse. L'examen microscopique d'une quarantaine de ces chancres révélait la présence de péritbèces de $N$. galligena seulement dans deux cas. La mise en culture de tissu infecté provenant de ces mêmes

${ }^{1}$ Département de Génie Forestier, Université Laval, Québec. 
chancres, pour y déceler la présence du champignon, ne fut guère plus heureuse à cause de nombreuses contaminations.

Au cours de l'été 1964, des observations effectuées dans la forêt expérimentale de Dudswell, comté de Compton, ont permis de décrire l'état de santé d'un bon nombre d'arbres. Ainsi, l'examen de la cime de 44 bouleaux jaunes montra que 29 d'entre eux avaient une couronne saine et que les 15 autres souffraient d'une mort en cime variant de 15 à 40 p. 100 . A la suite de l'abattage de 8 bouleaux jaunes dont 5 à cime affectée, nous avons pu constater que, chez ces derniers, la mort des branches et des rameaux était attribuable au développement d'un petit chancre ou d'une lésion sans callus sous l'écorce ou d'un groupe de chancres qui avaient coalisé (Fig. 1).

L'examen de 50 chancres ou lésions sans callus a révélé la présence de périthèces du champignon $N$. galligena sur 37 d'entre eux soit immédiatement, soit après avoir été gardés quelques jours dans une atmosphère saturée.

Sur les branches ayant un diamètre de $3 / 4$ à 1 pouce, nous avons observé des chancres circulaires avec un bourrelet cicatriciel bien développé (Fig. 2). Certains chancres ont annelé la tige et, le plus souvent, l'action combinée de plusieurs chancres a produit le même résultat. Sur les rameaux de moins de $1 / 2$ pouce, les chancres n'avaient pas atteint leur plein développement. Seule une tache déprimée (lésion) avait alors annelé la tige sans qu'aucun bourrelet ne se soit développé (Fig. 3). L'écorce, naturellement rouge verdâtre, était d'un brillant brun rougeâtre.

INOCULATIONS

Des inoculations effectuées à Duchesnay, à la suite des observations de 1959, ont permis de vérifier l'hypothèse que ces chancres et lésions résultaient de l'action du $N$. galligena. A cette fin, nous avons blessé et inozulé des branches de $3 / 4$ de pouce et des rameaux de $1 / 2$ pouce avec des gouttes d'une suspension de conidies.

TABLEAU 1

RESULTATS D'INOCULATIONS' ${ }^{2}$ DU $N$. galligena SUR DES BRANCHES ET DES RAMEAUX DE BOULEAU JAUNE ( $B$. alleghaniensis)

\begin{tabular}{|c|c|c|c|c|c|}
\hline Date & & & Inoculations & \multicolumn{2}{|c|}{ ResuItats } \\
\hline & & (Diam.) & (no.) & (no.) & (no.) \\
\hline \multirow{2}{*}{ Sept. 59} & Rameau & ( $1 / 2$ po.) & 4 & 1 & 3 \\
\hline & Branche & $\left(\begin{array}{ll}3 / 4 & \text { po. }\end{array}\right)$ & 4 & 2 & 0 \\
\hline \multirow[t]{2}{*}{ Mars. 60} & Rameau & $\left(\begin{array}{ll}1 / 2 & \text { po. }\end{array}\right)$ & 4 & 0 & 1 \\
\hline & Branche & ( $3 / 4$ po. $)$ & 4 & 2 & 0 \\
\hline \multirow[t]{2}{*}{ Juin 60} & Rameau & $\left(\begin{array}{ll}1 / 2 & \text { po. })\end{array}\right.$ & 4 & 0 & 2 \\
\hline & Branche & $\left(\begin{array}{ll}3 / 4 & \text { po. })\end{array}\right.$ & 4 & 3 & 0 \\
\hline
\end{tabular}

${ }^{1}$ Inoculations avec des gouttes d'une suspension de conidies sur des blessures légères faites au couteau.

A la suite de douze essais effectués sur des rameaux de bouleau jaune (Tableau 1), 6 ont produit une lésion sans callus (Fig. 4) alors qu'un seul chancre typique se développait. Dans tous les cas où il y eut lésion sans callus, la portion distale était flétrie et les tissus morts. Par ailleurs, sur les branches 

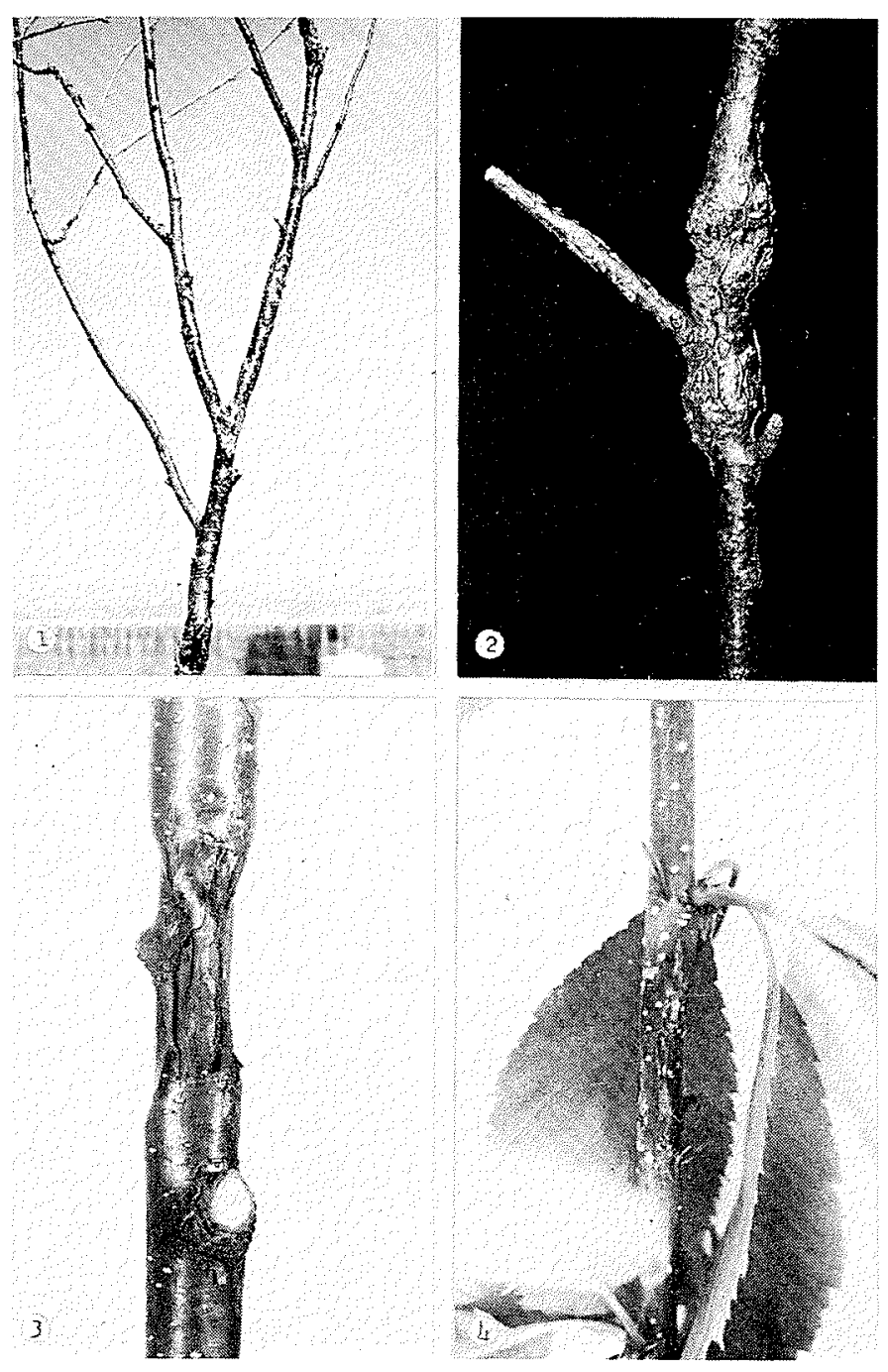

FIGURE 1. Nombreux chancres et lésions sans callus dus à l'action du Nectria galligena Bres. sur une branche de bouleau jaune.

FIGURE 2. Pecits chancres circulaires à bourrelets épais sur une branche de $3 / 4$ pouce de diamètre.

FIGURE 3. Lésion sans callus qui a annelé un rameau de $1 / 2$ pouce de diamètre.

FIGURE 4. Lésion sans callus qui s'est développée sur un rameau d'un diamètre inférieur à $1 / 2$ pouce de diametre à la suite d'une inoculation avec une suspension de conidies de Nectria galligena Bres. 
de $3 / 4$ pouce, les inoculations se transformaient en de véritables chancres et aucune lésion ne se développait. Les témoins inoculés à l'eau stérile se cicatrisèrent sans laisser de trace.

\section{Discussion}

Le champignon $N$. galligena peut attaquer les branches et les rameaux du bouleau jaune. Kienholz et Bidwell (1938), Grant et Spaulding (1932) l'avaient déjà rapporté mais certaines observations (Andrews, 1935) semblaient réduire l'importance du chancre nectrien sur les branches. Grâce aux inoculations, nous avons pu confirmer les observations des auteurs cités plus haut. De plus, les résultats confirment l'opinion de Grant et Spaulding (1932) à l'effet, que sur les branches de $3 / 4$ pouce et plus, une infection se développe normalement en un chancre, tandis que sur les rameaux de $1 / 2$ pouce et moins, l'infection annelle la tige et n'a pas le temps de permettre le plein développement du chancre; les bourrelets sont alors absents.

Les chancres qui apparaissent ainsi sur les branches des bouleaux jaunes sont moins caractéristiques que ceux qui se manifestent sur le tronc des mêmes arbres. Ils sont plutôt circulaires qu'ellipsoïdes et possèdent de très gros bourrelets. Sans doute, la dimension de la tige porteuse du chancre est-elle responsable de cette morphologie particulière.

Les réactions des arbres vivant dans des conditions identiques ne sont pas toujours les mêmes. Ainsi, certains arbres ne portaient aucun chancre de branche et aucune lésions de rameau, alors que l'arbre porteur en avait toujours un très grand nombre. Puisque les conditions d'environnement étaient similaires, nous supposons que les bouleaux jaunes ont des propriétés individuelles et que certains sont beaucoup plus sensibles que d'autres à l'action du $N$. galligena. D'ailleurs, on a pu démontrer l'existence de conditions différentes de résistance et de susceptibilité chez les arbres fruitiers (Viennot-Bourgin, 1949).

Il importe de noter l'importance des présentes observations en rapport avec les études sur le dépérissement du bouleau jaune. Du sol, l'aspect général des arbres affectés de nombreux chancres et lésions se confond facilement avec celui des arbres atteints de dépérissement. Il est donc possible qu'au cours d'études effectuées sur le développement et le progrès du dépérissement du bouleau jaune, certains observateurs aient confondu les deux problèmes. Sans doute, le nombre d'arbres affectés d'une mort en cime à cause de l'action du $N$. galligena est-il peu élevé. Mais le fait que de tels arbres soient groupés dans certaines forêts, comme à Dudswell, permet de supposer qu'un observateur puisse se méprendre en certaines circonstances. Enfin, les présentes observations suggèrent que tous les cas de mort en cime ne peuvent être imputés aux causes responsables du dépérissement généralisé du bouleau jaune.

\section{BIBLIOGRAPHIE}

ANDREWS, S. R. 1935. The incidence of Nectria canker in the sugar maple-beech-yellow birch type near Kane, Pennsylvania. Thesis for Master of Forestry degree. Yale School of forestry, $88 \mathrm{p}$.

GRANT, T. J. and P. SPAULDING. 1932. Avenues of entrance for canker-forming Nectrias of New England hardwoods. Phytopathology 29:351-358.

KIENHOLZ, R. and C. B. BIDWELL. 1938. A survey of disease and defects in Connecticut forests. Conn. Agr. Sta. Bul. 412:493-559.

VIENNOT-BOURGIN, G. 1949. Les champignons parasites des plantes cultivées. Tome I et II, Masson et Cie, Ed. Paris. 1851 p.

ZELLER, S. M. 1926. European canker of pomaceous fruit trees. Oreg. Coll. Agr. Exp. Sta. Bul. 222:52 p. 